\title{
Determinación de propiedades fisicoquímicas de propóleos provenientes de cinco especies de abejas sin aguijón de Norte de Santander-Colombia
}

\author{
Milena Silva Rojas; Alexandra Torres; Wolfgang Hoffmann \\ Grupo de Investigación Biocalorimetría. Facultad de Ciencias Básicas. Universidad de Pamplona.
}

\section{Resumen}

En el presente trabajo se realizó la determinación de las propiedades fisicoquímicas a muestras de propóleo recolectado por cinco especies de abejas sin aguijón: Melipona favosa favosa, Melipona fuscipes, Trigona (Frieseomelitta) nigra paupera, Trigona (Tetragosnisca) angustula, Scaptrotrigona sp. y la especie Apis mellifera, presentes en el Departamento de Norte de Santander- Colombia. Los análisis realizados son algunos de los establecidos en la norma rusa RST-RSFSR-317-77, la AOAC (Association of Official Analytical Chemists), y la Reglamentación del Ministerio de Agricultura de Brasil (Reglamento Técnico para fijar la identidad y calidad de propóleos). Las propiedades determinadas para el propóleo como materia prima fueron porcentaje de cera, índice de oxidación, determinación cualitativa de flavonoides, porcentaje de impurezas mecánicas, compuestos fenólicos, índice de yodo, cenizas, humedad, y solubilidad en etanol. El análisis del propóleo en solución se realizó para EEP (extracto de propóleo en etanol). Para la identificación de compuestos flavonoides se preparó un extracto hidroalcohólico. Los resultados mostraron que los propóleos producidos en esta región son de buena calidad y por tanto pueden ser comercializadas y distribuidos, presentando las siguientes variaciones respecto a cada parámetro analizado: a) propóleo como materia prima: porcentaje de cera (1-43,8\%), índice de oxidación (14,7-97 segundos), determinación cualitativa de flavonoides (positiva), porcentaje de impurezas mecánicas $(1,83$ $14,48 \%)$, compuestos fenólicos $(37,12-60,73 \%)$, índice de yodo $(49,39-79,31 \%)$, cenizas $(0,14-17,40 \%)$, humedad $(0,72-10,78 \%)$, y solubilidad en etanol $(0,41-5,80 \%)$. b) propóleo en solución: para EEP: índice de oxidación (1-5 segundos). Se confirmó la presencia de flavonoides y compuestos fenólicos en los extractos hidroalcohólicos.

Palabras clave: propóleo, abejas sin aguijón, propiedades fisicoquímicas.

\section{Determination of physicochemical properties of propolis of five species of stingless bees from Norte de Santander-Colombia}

\section{Abstract}


This work shows the physicochemical analysis of propolis samples of five species of stingless bees: Melipona favosa favosa, Melipona fuscipes, Trigona (Frieseomelitta) nigra paupera, Trigona (Tetragosnisca) angustula, Scaptrotrigona sp. , in Departamento Norte de Santander, Colombia. The analyses performed are those proposed in the Russian law RST-RSFSR-31777, the AOAC (Association of Official Analytical Chemists) and the Reglamentación del Ministerio de Agricultura de Brasil (Technical Regulations for setting the identity and quality of propolis). The properties determined for the raw propolis were the percentage of wax, oxidation index, qualitative determination of flavonoids, mechanical impurities, phenolic compounds, iodine index, ash, moisture, and solubility in ethanol. The analysis of oxidation index for propolis extracts, were performed to EEP (ethanol extract). For the identification of flavonoid compounds we prepared a hydroalcoholic extract. Our results showed that the propolis produced in this region are of good quality and therefore can be marketed and distributed, featuring the following variations for each parameter analyzed: a) raw propolis as: percentage of wax (1-43,8\%), oxidation index (14,7-97 seconds), qualitative determination of flavonoids (positive), mechanical impurities (1,83-14,48\%), phenolic compounds (37,12$60,73 \%)$, iodine index $(49,39-79,31 \%)$, ash $(0,14-17,40 \%)$, moisture $(0,72-10,78 \%)$, and solubility in ethanol $(0,41-5,80 \%)$. b), propolis extracts: for EEP: oxidation index (1-5 seconds). All hydroalcoholic extracts have flavonoids and phenolic compounds.

Keywords: propolis, stingless bees, physicochemical properties.

*Para citar este artículo: Silva Rojas M; Torres A; Hoffmann W. Determinación de propiedades fisicoquímicas de propóleos provenientes de cinco especies de abejas sin aguijón de Norte de Santander-Colombia. Revista Bistua.2016.14(1):03-16

+ Autor para el envió de correspondencia y la solicitud de las separatas: Alexandra Torres. Grupo de Investigación Biocalorimetría. Facultad de Ciencias Básicas. Universidad de Pamplona.

email:datorres@unipamplona.edu.co 
05

\section{Introducción}

Las abejas hacen parte de un grupo de insectos altamente distribuidos en las áreas tropicales y subtropicales del mundo; su importancia ecológica y económica radica en su papel como polinizadores de numerosas plantas y en el aporte que brindan al hombre con la producción de miel, cera y propóleo entre otros productos ${ }^{1}$.En Colombia, se cuenta con más de 600 especies de abejas nativas, sin embargo la industria gira alrededor de una sola especie foránea famosa por la producción de miel, denominada Apis mellifera (conocida como abeja africanizada). Las abejas sin aguijón ofrecen variados productos, los cuales suelen ser aprovechados por los meliponicultores como: la miel, el polen, la cera, y el propóleo, que se caracterizan popularmente por poseer propiedades medicinales $^{2}$. El propóleo, es una sustancia elaborada por las abejas que está compuesta por resinas de diferentes plantas, y que una vez colectadas son enriquecidas con secreciones salivares y enzimáticas producidas por las abejas. Uno de sus principales usos dentro de la colmena consiste en su sellamiento hermético para impedir infecciones o entrada de enemigos dentro de ella. Su coloración puede ser verde pardo, castaño o incluso casi negro, dependiendo de su origen botánico. Tiene sabor acre, frecuentemente amargo, y olor agradable y dulce, y cuando se quema, exhala una fragancia de resinas aromáticas, conocida desde hace siglos por las innumerables propiedades que posee, gracias a su compleja composición química obtenida de la flora de donde las abejas lo recolectan ${ }^{3,}{ }^{4}$.Desde la antigüedad, los sacerdotes egipcios utilizaban el propóleo de manera medicinal y en la momificación de cadáveres para su conservación. Tiempo después fueron los sabios griegos quienes le dieron el nombre con el que se conoce en la actualidad: Pro- defensa y Polis- ciudad, es decir, "defensa de la ciudad" (defensa de la colmena). El propóleo contiene flavonoides a los que se le han atribuido propiedades terapéuticas. Además, de estos compuestos, contiene bálsamos, cera de abeja, aceites esenciales, polen y diversos minerales entre ellos: aluminio, plata, bario, boro, cromo, cobalto, estaño, hierro y muchos otros. También contiene provitamina $A$ y vitaminas del grupo B, especialmente B3. Entre la gran variedad de propiedades medicinales que posee el propóleo se encuentra su capacidad cicatrizante, anestésica, antiinflamatoria, antibacteriana, antimicótica, antiviral, vaso protectora y antitumoral. ${ }^{5}$ Las propiedades curativas del propóleo se relatan a lo largo del antiguo testamento, las propiedades antimicrobianas del propóleo se atribuyen a su alto contenido en flavonoides. ${ }^{6}$ El propóleo también fue reconocido por otros protagonistas de 
las antiguas civilizaciones: los incas posteriores de limpieza, emplearon el propóleo como agente antipirético y las farmacopeas del siglo XVII lo designaban ya como una drogaoficial y en Europa afianzo su popularidad entre los siglos XVII y el siglo XX a causa de su actividad antibacteriana;pero cuando se descubrió la penicilina y demás antibióticos el propóleo fue dejado a un lado. Actualmente, se están realizando muchas investigaciones ya que los nuevos antibióticos además de presentar reacciones adversas muestran una marcada resistencia a algunos microorganismos ${ }^{7}$.La demanda de propóleo se ha incrementado debido al conocimiento a nivel mundial de sus múltiples propiedades; tomando gran fuerza en el mercado como materia prima empleada en la solución de problemas tanto en el área alimenticia como farmacológica, incorporado a través de productos como: caramelos, tinturas, chicles, jabones, lociones, ungüentos, pastillas, sales de baño, vinos, polvos, champús, cremas, bebidas, etc. Colombia no produce propóleo en cantidades suficientes para abastecer su demanda interna y por lo tanto, se convierte en un importador neto de este producto tanto en forma natural como procesada. El principal exportador de propóleo es Estados Unidos, seguido por Alemania y Francia. 8

El propóleo producido en Colombia proviene en su totalidad de las abejas Apis mellifera, pero debido a que no existe ninguna normatividad estatal en cuanto a su recolección y tratamientos

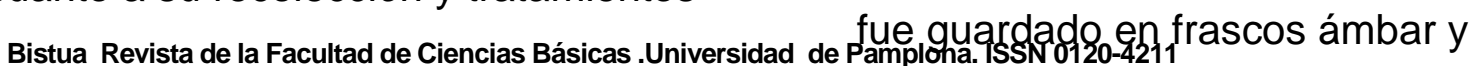

Muestreo. Se tomaron muestras de propóleo de diferentes partes del nido de seis especies: Apis mellifera, Trigona (Frieseomelitta) nigra paupera, Scaptrotrigona sp., Melipona favosa, Melipona fuscipes, y Trigona (Tetragonisca) angustula. Las muestras fueron tomadas por el método de raspado directamente de colmenas racionales localizadas según lo estipulado en la tabla 1. El material 
07

trasladado al laboratorio de Biocalorimetría de la Universidad de Pamplona donde se almacenó protegido de la luz y bajo congelación a $-18^{\circ} \mathrm{C}$.

Preparación y acondicionamiento de las muestras

La preparación de las muestras para su análisis comprendió los siguientes tratamientos:

\section{a) Propóleo como materia prima pura:}

Debido a que la mayoría de las muestras presentan consistencia blanda y pegajosa, su manipulación es muy difícil, por ello se mantuvieron en congelación, y previo a su análisis se trituraron y homogenizaron con una moledora para café marca Simply.

\section{b) Propóleo en solución:}

Se tomaron 4,5 g de propóleo crudo de cada especie y se disolvieron en $90 \mathrm{~mL}$ de etanol del 96\% v/v (Merck) y se maceraron protegidos de la luz durante un periodo de tiempo de 15 días. Después de éste tiempo los extractos se filtraron, utilizando para ello papel de filtro Schleicher \& Schuell 595, ф 125 $\mathrm{mm}$. El filtrado se guardó en frascos ámbar $\mathrm{a}-18^{\circ} \mathrm{C}$ hasta su uso.

\section{Análisis fisicoquímico}

Se determinaron las propiedades fisicoquímicas por triplicado de: a) muestras de propóleos como materia prima (porcentaje de cera, índice de oxidación, presencia de flavonoides, porcentaje de impurezas mecánicas, compuestos fenólicos, índice de yodo, cenizas, humedad, y solubilidad en etanol) y b) extracto etanólico de propóleo (índice de oxidación, presencia de flavonoides y compuestos fenólicos). Los espectros de absorción UV se midieron en un espectrofotómetro UV-2401 PC, Shimadzu, dotado con el software UV Probe 2.01.

La metodología utilizada para la determinación de propiedades fisicoquímicas es la descrita en la Norma Rusa ${ }^{20}$, la AOAC (Association of Official Analytical Chemists) ${ }^{21}$, y la Reglamentación del Ministerio de Agricultura de Brasil 22. Los resultados fueron evaluados de acuerdo con las normas anteriores teniendo en cuenta que en Colombia no existe legislación.

Con el objetivo de determinar si se encontraban diferencias significativas entre y dentro de las especies, se consideraron en conjunto todos los datos de las muestras que fueron sometidos al análisis estadístico ANOVA, seguido por Bonferroni posthoc tests con un nivel de significancia de $5 \%(p<0,05)$, mediante el uso del paquete estadístico de distribución libre: paleontological statistic software package for education and data analysis (PAST).

\section{Propóleo como materia prima}

Los resultados obtenidos de las propiedades fisicoquímicas de los propóleos analizados como materia prima, se muestran en la tabla 2.

\section{Porcentaje de cera}


08

El porcentaje de cera de las especies analizadas varió entre $1,01 \%$ y $43,83 \%$, siendo más alto el de la especie Apis mellifera, estos resultado concuerdan con lo reportado por Araujo et al. (2016) para las especies $M$. scutellaris y $M$. fasciculata en Brasil. En cuanto a las especies de abejas sin aguijón los valores más altos los presentan las especies Trigona (Tetragonisca) angustula y Scaptrotrigona sp. Las dos especies del género Melipona presentan un contenido de cera bastante bajo.

Con respecto al análisis estadístico se encontraron diferencias significativas entre $A$. mellifera con $M$. favosa, $M$. fuscipes y $T$. Frieseomelitta. La especie $T$. angustula con $M$. favosa, $M$. fuscipes y $T$. Frieseomelitta. La especie Scaptotrigona sp. presentó diferencias significativas con todas las demás. Al comparar especies de la misma tribu los valores obtenidos reflejan que entre las dos especies del género Melipona no se encontraron diferencias significativas, mientras que las tres especies del género Trigona presentaron diferencias significativas entre ellas.

La norma rusa (basada en propóleo de Apis mellifera) establece como máximo contenido de cera $30 \%$, las especies Apis mellifera y Trigona (Tetragonisca) angustula presentaron mayores valores. $\mathrm{Si}$ se tiene en cuenta éste valor como criterio de calidad, el potencial de comercialización del propóleo obtenido de las abejas sin aguijón es promisorio.

Índice de oxidación

Bistua Revista de la Facultad de Ciencias Básicas .Universidad de Pamplona. ISSN 0120-4211
El índice de oxidación de las muestras varía entre 14,7 seg y $97,0 \mathrm{seg}$, siendo en orden ascendente: $M$. fuscipes < Scaptrotrigona sp. $<T$. Frieseomelitta $<A$. mellifera $<T$. angustula $<M$. favosa. Se encontraron diferencias significativas de $M$. fuscipes con $A$. mellifera y $M$. favosa. También $T$. Frieseomelitta presenta diferencias con $M$. favosa. La especie $T$. angustula con $M$. fuscipes y $T$. Frieseomelitta.

La especie Scaptotrigona sp., presentó diferencias significativas con $A$. mellifera, $M$. favosa y $T$. angustula. Los resultados muestran que hay diferencias significativas entre las especies pertenecientes a los mismos géneros. Según algunos autores Souza et al. (2002) Graças et al. (2002) y Evandro et al. (2002) las variaciones pueden asociarse con la presencia de compuestos como el ácido caféico, ésteres, sesquiterpenoides, nerodiol y cinamato que le confieren propiedades diferentes a los propóleos y que podrían modificar la respuesta esperada del índice de oxidación.

Adicionalmente, ésta propiedad sirve para verificar propiedades antioxidantes del propóleo. Según la norma rusa como referencia se suelen considerar valores hasta de 22 segundos. Las especies Melipona fuscipes y Scaptrotrigona sp.fueron las únicas dentro de este rango.

Determinación cualitativa de flavonoides.

La presencia de compuestos flavonoides de todas las muestras 
estudiadas fue positiva (Todas las muestras cumplen con la Norma Rusa). Los flavonoides son importantes para las plantas $y / 0$ a sus flores ya que le proporcionan color y olor para atraer polinizadores. Tanto en animales como en seres humanos las investigaciones realizadas muestran que tienen propiedades medicinales como antioxidantes, anticancerosas, antiinflamatorias, etc. por lo que se les considera los compuestos insignia del propóleo. Debido a que los flavonoides del propóleo provienen exclusivamente de las plantas, la determinación cualitativa puede usarse como criterio de adulteración del propóleo.

\section{Porcentaje de impurezas mecánicas}

El porcentaje de impurezas mecánicas de las muestras varía entre $1,83 \%$ y $14,48 \%$, siendo en orden ascendente: M. favosa $<T$. Frieseomelitta $<A$. mellifera $<T$. angustula $<M$. fuscipes $<$ Scaptrotrigona sp. Se encontraron diferencias significativas de $M$. fuscipes con $M$. favosa. También $T$. frieseomelitta presenta diferencias con M. fuscipes. La especie Scaptotrigona sp., presentó diferencias significativas con $A$. mellifera, $M$. favosa, $T$. Frieseomelitta y $T$. angustula. La especie $T$. angustula no presentó diferencias significativas con ninguna especie. La especie que presentó el valor más grande fue Scaptotrigona sp., lo que está directamente relacionado con las diferencias significativas que presentó ésta especie con respecto a las demás. Por el contrario los valores obtenidos tanto de porcentaje de impurezas mecánicas como en el

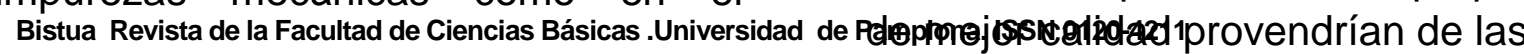
proveniente de ésta especie es muy similar al de las demás.

Las impurezas mecánicas en el propóleo pueden estar constituidas por partes de abejas, partes de plantas, madera, tierra entre otras. Debido a esto un valor alto de éste parámetro hace que el propóleo sea catalogado como de baja calidad. La norma rusa establece como valor máximo $20 \%$, lo que permite catalogar a todas las muestras de las diferentes especies analizadas en el presente trabajo como de buena calidad.

\section{Porcentaje de compuestos fenólicos}

Los valores de porcentaje de compuestos fenólicos para las muestras analizadas se encuentran entre $37,12 \%$ y $60,73 \%$., siendo en orden ascendente: $M$. fuscipes $<A$. mellifera $<M$. favosa $<T$. angustula $<$ T. Frieseomelitta $<$ Scaptrotrigona sp

El análisis de varianza mostró que no existen diferencias significativas entre la cantidad de compuestos fenólicos presentes en las muestras de própoleos de las cinco especies de abejas sin aguijón y $A$. Mellifera. Los compuestos fenólicos al igual que los compuestos flavonoides del propóleo provienen únicamente de las plantas, y tienen múltiples aplicaciones medicinales para los seres humanos. Los valores obtenidos del porcentaje de compuestos fenólicos de las muestras analizadas se encuentran por encima del valor mínimo permitido $(30 \%)$ en la Norma Rusa. Teniendo en cuenta éste parámetro los propóleos angustula indican que el propóleo 
También de $M$. fuscipes con $M$. favosa

especies Scaptrotrigona sp. y Trigona Frieseomelitta.

\section{Índice de yodo}

Según lo reportado en la tabla 2 , se encontraron diferencias significativas de M. favosa con $A$. mellifera. También $T$. angustula con $A$. mellifera. No se presentaron diferencias significativas entre especies del mismo género melipona ni las del género trigona. El índice de yodo es una medida del grado de insaturaciones de los componentes de una grasa.

Debido a que los aceites vegetales son grasas insaturadas, el porcentaje de yodo permite conocer que tan rico en éstos compuestos es un determinado propóleo. Los valores obtenidos del porcentaje de índice de yodo de las muestras analizadas se encuentran por encima del valor mínimo permitido $(35 \%)$ en la norma rusa, según esto, el propóleo proveniente de la especie $A$. mellifera podría considerarse como el de mejor calidad.

\section{Determinación de cenizas}

Los valores de porcentaje de cenizas para las muestras analizadas se encuentran entre $0,14 \%$ y $17,40 \%$, este último valor difiere grandemente de lo encontrado en Brasil para las especies $M$. scutellaris y $M$. fasciculata 23 .Los valores en orden ascendente son: $M$. favosa $<T$. Frieseomelitta $<T$. angustula = $A$. mellifera < Scaptrotrigona sp. < M. fuscipes. Se encontraron diferencias significativas entre $T$. angustula y $A$. mellifera. y A. mellifera. M. fuscipes presentó diferencias significativas con $T$. Frieseomelitta, T. angustula y Scaptotrigona sp.

Teniendo en cuenta, los géneros a los que pertenecen las especies, no se presentaron diferencias significativas entre las especies pertenecientes al género Trigona. Las muestras provenientes de las especies $T$. angustula, A. mellifera, $M$. favosa, $T$. Frieseomelitta, y Scaptotrigona sp., poseen un bajo contenido de cenizas lo que indica que presentan pocos residuos inorgánicos, es decir, presentan bajo contenido de minerales. Comparando los valores obtenidos en este trabajo con la Reglamentación del Ministerio de Agricultura de Brasil (Reglamento Técnico para fijar la identidad y calidad de propóleos) que establece como porcentaje máximo de cenizas el 5\%, las especies mencionadas anteriormente se encuentran dentro del intervalo.

La especie $M$. fuscipes presenta un valor superior al aceptado por la reglamentación de Brasil. El aspecto del residuo obtenido en la prueba, evidencia la diferencia en los constituyentes del propóleo de esta especie con respecto a las demás, por lo que podría asumirse que esta especie recolecta mayor cantidad de agregados minerales provenientes posiblemente del suelo ya que a simple vista este propóleo tiene apariencia terrosa.

\section{Determinación de humedad}


blancas y verdosas; además de que se

Los valores de porcentaje de humedad para las muestras analizadas se encuentran entre $0,72 \%$ y $10,78 \%$., siendo en orden ascendente: $M$. favosa $<M$. fuscipes < Scaptrotrigona sp. < A. Mellifera $<T$. Frieseomelitta $<T$. angustula. Se encontraron diferencias significativas entre $M$. favosa y $A$. mellifera. También de $T$. Frieseomelitta con $M$. favosa. $T$. angustula presentó diferencias significativas con $M$. fuscipes, $M$. favosa y $A$. mellifera.

Adicionalmente, $T$. angustula y Scaptotrigona sp. presentaron entre ellas diferencias significativas. No se presentaron diferencias significativas entre las especies pertenecientes al género Melipona.

Los valores de porcentaje de humedad de todas las especies a excepción de la especie $T$. angustula, están dentro del porcentaje máximo de humedad del $8 \%$, según la Reglamentación del Ministerio de Agricultura de Brasil. La consistencia observada en las muestras está relacionada con el contenido de agua, la de mayor contenido en humedad $T$. angustula mostró una consistencia más blanda en comparación a las demás haciendo más difícil el manejo de la misma. En contraste, la muestra obtenida de la especie $M$. favosa, puesto que esta no tenía que ser congelada para poder pulverizarla. La humedad excesiva en el propóleo favorece el desarrollo de algunas especies de mohos y levaduras en la superficie del mismo, lo que se manifiesta por la presencia de capas pueden producir fermentaciones que a su vez generan productos no deseados, se pueden generar toxinas perjudiciales para la salud. Teniendo en cuenta lo anterior, los propóleos analizados en el presente trabajo no tendrían éste problema.

\section{Solubilidad en etanol}

Los valores de porcentaje de solubilidad en etanol para las muestras analizadas se encuentran entre $0,41 \%$ y $5,80 \%$., siendo en orden ascendente: $T$. angustula $<A$. mellifera $<M$. fuscipes < T. Frieseomelitta < Scaptrotrigona sp. < M. favosa. Se encontraron diferencias significativas entre $M$. favosa y $A$. mellifera. También de T. Frieseomelitta con M. favosa. $T$. angustula presentó diferencias significativas con $T$. Frieseomelitta y $M$. favosa. Adicionalmente, Scaptotrigona sp., presentó diferencias significativas con todas las especies a excepción de $T$. Frieseomelitta. También entre $M$. favosa y $M$. fuscipes se presentaron diferencias.

Los valores del porcentaje de solubilidad en etanol para todas las muestras de propóleos analizadas están muy por debajo del mínimo establecido (35\%) en la Reglamentación del Ministerio de Agricultura de Brasil. Desafortunadamente, en el caso de las abejas sin aguijón, no existe ningún estudio en Colombia con el cual comparar. Para la especie A. mellifera se han reportado valores de propóleos provenientes de diferentes regiones del 
país en donde los porcentajes oscilan entre $7 \%$ a $94 \%$. A pesar de que el propóleo tiene bajo porcentaje de sustancias solubles en etanol éste presenta una relativamente rápida decoloración de la solución de permanganato, indicando que posiblemente están presentes compuestos de naturaleza fenólica, o con grupos funcionales oxidables (hidroxilos, instauraciones entre otras), los cuales pueden exhibir actividad biológica.

\section{Propóleo en solución}

Los valores del índice de oxidación (tiempo de decoloración de la solución del $\mathrm{KMnO}_{4}$ medido en segundos) de los extractos de las diferentes muestras de propóleo son bajos y están dentro del límite establecido por la norma rusa de máximo 22 segundos. No se encontraron diferencias para cada especie con relación a cada uno de los extractos.Todas las muestras presentaron espectros de absorción semejantes con una banda amplia a longitudes de onda máxima de $290 \mathrm{~nm}$, con lo cual se comprueba la presencia de compuestos fenólicos y flavonoides. Adicionalmente, la mayor absorbancia se presenta para la especie Melipona favosa $(1,66)$ y la más baja para la especie Scaptotrigona sp. $(0,23)$ estas diferencias pueden corresponder al diferente origen botánico y geográfico de los mismos.

\section{Conclusiones}

La determinación de las propiedades fisicoquímicas del propóleo permitió realizar un análisis preliminar acerca de los principales parámetros y compuestos que hacen parte de los lineamientos contemplados en los estándares de calidad, permitiendo así conocer y aprovechar las ventajas potenciales de este producto.

En este trabajo se lograron establecer semejanzas y diferencias entre las especies consideradas por medio del análisis ANOVA, reconociendo que en la mayoría de los parámetros examinados existen diferencias significativas entre las especies estudiadas. Un caso particular se manifiesta en Scaptotrigona sp., donde dicha especie muestra diferencias significativas con casi todas las especies en cuatro parámetros (porcentaje de cera, índice de oxidación, impurezas mecánicas y solubilidad en etanol) sin mostrar relación alguna con las especies del mismo sitio de recolección. Los parámetros donde se encontraron diferencias significativas con respecto a una especie (como mínimo) de abejas sin aguijón fueron: porcentaje de cera, índice de oxidación, índice de yodo, cenizas, humedad, impurezas mecánicas y solubilidad en etanol.

En cuanto a la estabilidad frente la temperatura ambiente de las muestras analizadas, se observó que casi todas poseían una apariencia resinosa con excepción del propóleo recolectado por la especie Melipona fuscipes que tenía 
Todas las muestras analizadas

aspecto terroso y de acuerdo a los resultados de la composición en cenizas dicha especie fue la que presentó mayor contenido de estas, evidenciando una cantidad relativamente alta de residuos inorgánicos provenientes probablemente del suelo. De la cantidad de humedad se puede decir que a medida que ésta se incrementa, la muestra se hace más gomosa.

De acuerdo con los parámetros evaluados conforme a la Norma Rusa y a la Reglamentación del Ministerio de Agricultura de Brasil, la muestra de propóleo de la especie Apis mellifera es de buena calidad con algunas excepciones en cuanto al contenido de cera, índice de oxidación y solubilidad en etanol. En cuanto a las muestras provenientes de las especies de abejas sin aguijón se puede decir que también cumplen con los valores establecidos para cada uno de los parámetros contemplados en esta investigación, presentando algunas excepciones en el contenido de cera para la especie $T$. angustula, en el índice de oxidación las variaciones se presentaron para las especies $M$. favosa, T. Frieseomelitta y $T$. angustula, en el contenido de humedad para $T$. angustula, en el contenido de cenizas la excepción fue la especie $M$. fuscipes . Finalmente, en el parámetro de solubilidad en etanol ninguna especie se acercó al valor mínimo permitido probablemente por la naturaleza de los compuestos presentes en las muestras. contienen flavonoides, componentes que generalmente despiertan el mayor interés para el posible uso medicinal del propóleo, razón por la cual, se requiere con urgencia no solamente determinar las propiedades biológicas de los mismos y los compuestos responsables de la actividad, sino, recolectar información de las diferentes especies provenientes de diferentes sitios para establecer una normatividad que conduzca a fortalecer la incipiente industria nacional.

\section{Agradecimientos}

Los autores agradecen al laboratorio de Control de Calidad de la Universidad de Pamplona por facilitar el empleo de equipos y de algunos reactivos.

\section{Referencias bibliogràficas}

1.-Abrahamovich H, Díaz N, Lucia M. Identificación de las "abejas sociales" del género Bombus (Hymenoptera, Apidae) presentes en la Argentina: clave pictórica, diagnosis, distribución geográfica y asociaciones florales. Rev Fac Agro. 2007; 106 (2): 165-176.

2.-Enríquez ME, Obiols CL, Dardón MJ. Manual: Biología y Reproducción de abejas nativas sin aguijón. Guatemala: Editorial Universidad de San Carlos de Guatemala (USAC); 2006.

3.-Value-added products from beekeeping. R. Krell Editor. Food and Agriculture Organization of the United Nations Rome 1996. Disponible en http://www.fao.org/docrep/w0076e/w0076e 00.htm\#con

4.-Salatino A, Weinstein E, Negri G, Message D. Origin and Chemical Variation 
14

of Brazilian Propolis. eCAM. 2005; 2 (1): 33-38.

5.-Kumazawa S, Hayashi K, Kajiya K, Ishii T, Hamasaka T, Nakayama T. Studies of the constituents of Uruguayan propolis. J Agric Food Chem. 2002; 50 (17) : 47774782.

6.-Cushnie TP, Lamb AJ. Antimicrobial activity of flavonoids. Int $\mathrm{J}$ Antimicrob Agents. 2005; 26 (5): 343-356.

7.-Adcock $\mathrm{H}$. Pharmageddon: is it too late to tackle growing resistance to anti-infectives? Pharm J. 2002; 269: 599-600.

8.-http://www.proargex.gob.ar [actualizado 27 octubre 2010; citado 5 febrero 2016]. Disponible en: http://www.proargex.gob.ar/index.php/infode-mercado/26-estudios-y-perfiles-demercado/490-estudio-de-mercado-depropoleos-procesados

9.-Viloria JD, Gil JH, Durango DL, García CM. Caracterización fisicoquímica del propóleo de la región del bajo cauca antioqueño (Antioquia, Colombia). Biotec Sec Agrop Agroind. 2012; 10 (1): 77 - 86.

10.- Rodríguez $Y$, Sánchez-Catalán $F$, Rojano B, Durango D, Gil J, Marín-Loaiza J. Caracterización fisicoquímica y evaluación de la actividad antioxidante de propóleos recolectados en el Departamento del Atlántico, Colombia. Rev U.D.C.A Actu Div Cient. 2012; 15 (2): $303-311$.

11.-Ortega NS, Benitez N, Cabezas FA. Actividad antibacteriana y composición cualitativa de propóleos provenientes de dos zonas climáticas del Departamento del Cauca. Biotec Sec Agrop Agroind. 2011; 9 (1) : 8-16.
12.-Gómez J, Peña N, Pérez C, Gutiérrez $\mathrm{C}$, Suarez $\mathrm{H}$. Evaluación por dos métodos in vitro de actividad antimicrobiana de propóleos frente a algunos microorganismos de interés alimentario. Rev Fac Nal Agr Medellín. 2014; 67 (2): 131-134.

13.-Sanabria A, Cardenas LA, Parroquiano ML. Actividad antimicrobiana y examen fitoquímico preliminar de siete angiospermas y una muestra de propóleo. Rev Colom Cien Quím-Farma. 2002; 31 (1): 36-42.

14.-Ferreira EM, Guzmán D, Figueroa J, Tello J, De Oliveira D. Caracterización antimicrobiana y fisicoquímica de propóleos de Apis mellifera L. (Hymenoptera: Apidae) de la Región Andina Colombiana. Acta Biol Colomb. 2011; 16 (1): 175-184.

15.-Palomino LR, García CM, Gil JH, Rojano BA, Durango DI. Determinación del contenido de fenoles y evaluación de la actividad antioxidante de propóleos recolectados en el Departamento de Antioquia (Colombia). VITAE, Rev Fac Quim Farm. 2009; 16 (3) : 388-395.

16.-Palomino LR, Martínez JP, García CM, Gil JH, Durango DL. Caracterización fisicoquímica y actividad antimicrobiana del propóleos en el Municipio de La Unión (Antioquia, Colombia) Rev Fac Nal Agr Medellín. 2010; 63 (1): 5373-5383.

17.-Talero C, Hernández D, Figueroa J. Calidad microbiológica de propóleo crudo y sólidos solubles de extractos de propóleos de Apis mellifera en Colombia, Rev. Med. Vet. Zoot. 2012; 59 (2): 109118.

18.-Figueroa J, Salcedo J, Aguas $\mathrm{Y}$, Olivero R, Narvaez G. Recubrimientos comestibles en la conservación del mango y aguacate, y perspectiva, al uso del 
15

propóleo en su formulación. Rev Colom Cienc Anim. 2011; 3 (2): 386-400.

19.-Barrera E, Gil M, García CM, Durango $\mathrm{DL}$, Gil JH. Empleo de un recubrimiento formulado con propóleos para el manejo poscosecha de frutos de papaya (Carica papaya L. cv. Hawaiana). Rev Fac Nal Agr. Medellín. 2012; 65(1): 6497-6506.

20.-Russian Regional Standards RSFSR Norma Rusa RS-RSFR-317-77. 1977. [citado: 5 de febrero 2016]. Disponible en: http://www.api-

guia.com.ar/propoleos/metodos-

analiticos.htm

21.-A.O.A.C. Association of Official Analytical Chemists A.O.A.C. Official Methods of Analyisis.15th Edition, Washington D.C.1990.

22.-MAPA-MINISTÉRIO da agricultura, pecuária e abastecimento, BRASIL. Instrução Normativa no 3 , Anexo VII: Regulamento de identidade e qualidade de extrato de própolis; 2001.

23.-Araújo $K$, Júnior $J$, Sato $M$, Finco $F$, Soares I, Barbosa R, Alvim T, Ascêncio S, Mariano S. Physicochemical properties and antioxidant capacity of propolis of stingless bees (Meliponinae) and Apis from two regions of Tocantins, Brazil. Acta Amaz. 2016; 46(1): $61-68$.
Tabla 1. Sitios de muestreo

\begin{tabular}{|c|c|c|}
\hline Especie & Ubicación & $\begin{array}{l}\text { Altitud } \\
\text { (m.s.n.m.) }\end{array}$ \\
\hline Apis mellifera & $\begin{array}{l}\text { Finca Villa Marina } \\
\text { (Vereda Matajira) }\end{array}$ & 1.171 \\
\hline $\begin{array}{l}\text { Scaptrotrigona } \\
\text { sp. }\end{array}$ & $\begin{array}{l}\text { Finca Villa } \\
\text { Marina(Vereda Matajira) }\end{array}$ & 1.171 \\
\hline $\begin{array}{l}\text { Melipona } \\
\text { favosa }\end{array}$ & $\begin{array}{l}\text { km } 5 \text { vía Cúcuta - Puerto } \\
\text { Santander }\end{array}$ & 302 \\
\hline $\begin{array}{l}\text { Melipona } \\
\text { fuscipes }\end{array}$ & $\begin{array}{l}\text { Km } 13 \text { vía Pamplona - } \\
\text { Cúcuta (Vereda San } \\
\text { Rafael) }\end{array}$ & 1.639 \\
\hline $\begin{array}{l}\text { Trigona } \\
\text { angustula }\end{array}$ & Carmen de Tonchalá & 311 \\
\hline $\begin{array}{l}\text { Trigona nigra } \\
\text { paupera }\end{array}$ & $\begin{array}{l}\text { Finca Villa Marina } \\
\text { (Vereda Matajira) }\end{array}$ & 1.171 \\
\hline
\end{tabular}

Tabla 2. Propiedades fisicoquímicas de propóleos como materia prima. Los datos muestran la desviación estándar de tres muestras

\begin{tabular}{|c|c|c|c|c|}
\hline Especie & $\begin{array}{l}\text { Cera } \\
\%\end{array}$ & $\begin{array}{c}\text { Índice } \\
\text { oxidación } \\
\text { (segundos) }\end{array}$ & $\begin{array}{l}\text { Presencia } \\
\text { flavonoides }\end{array}$ & $\begin{array}{c}\text { Impurezas } \\
\text { mecánicas } \\
\%\end{array}$ \\
\hline M. favosa & $1,01 \pm 0,46$ & $97 \pm 2,4$ & + & $1,83 \pm 0,53$ \\
\hline M. fuscipes & $5,02 \pm 0,14$ & $14,7 \pm 0,4$ & + & $10,13 \pm 0,18$ \\
\hline $\begin{array}{l}\text { T. nigra } \\
\text { paupera }\end{array}$ & $1,29 \pm 0,35$ & $32 \pm 5,3$ & + & $34,00 \pm 0,41$ \\
\hline T. angustula & $34,74 \pm 1,32$ & $96 \pm 24,2$ & + & $5,32 \pm 0,05$ \\
\hline $\begin{array}{l}\text { Scaptrotrigona } \\
\text { sp. }\end{array}$ & $22,71 \pm 4,36$ & $20,7 \pm 0,9$ & + & $14,48 \pm 2,07$ \\
\hline Apis mellifera & $\begin{array}{c}43,83 \pm \\
5,22\end{array}$ & $93,67 \pm 24,4$ & + & $5,08 \pm 0,54$ \\
\hline
\end{tabular}




\begin{tabular}{|l|c|c|c|c|c|}
\hline \multicolumn{1}{|c|}{ Especie } & $\begin{array}{c}\text { Compuestos } \\
\text { fenólicos } \\
\%\end{array}$ & $\begin{array}{c}\text { Índice de } \\
\text { yodo } \\
\%\end{array}$ & $\begin{array}{c}\text { Cenizas } \\
\%\end{array}$ & $\begin{array}{c}\text { Humedad } \\
\%\end{array}$ & $\begin{array}{c}\text { Solubilidad } \\
\text { en etanol } \\
\%\end{array}$ \\
\hline M. favosa & $45,38 \pm 3,92$ & $49,82 \pm 2,64$ & $0,14 \pm 0,04$ & $0,72 \pm 0,16$ & $5,80 \pm 0,41$ \\
\hline M. fuscipes & $37,12 \pm 2,29$ & $60,05 \pm 2,26$ & $17,40 \pm 3,87$ & $3,52 \pm 0,1$ & $1,64 \pm 0,08$ \\
\hline $\begin{array}{l}\text { T. nigra } \\
\text { paupera }\end{array}$ & $55,93 \pm 8,60$ & $64,89 \pm 3,45$ & $0,45 \pm 0,07$ & $5,44 \pm 0,18$ & $2,70 \pm 0,37$ \\
\hline T. angustula & $47,09 \pm 2,64$ & $49,39 \pm 3,66$ & $0,90 \pm 0,01$ & $10,78 \pm 2,56$ & $0,41 \pm 0,20$ \\
\hline $\begin{array}{l}\text { Scaptrotrigona } \\
\text { sp. }\end{array}$ & $60,73 \pm 0,04$ & $58,81 \pm 0,06$ & $3,66 \pm 0,16$ & $3,66 \pm 0,13$ & $3,18 \pm 0,08$ \\
\hline Apis mellifera & $39,99 \pm 1,03$ & $79,31 \pm 6,61$ & $0,90 \pm 0,01$ & $4,19 \pm 0,12$ & $1,30 \pm 0,04$ \\
\hline $\begin{array}{l}\text { Límite de } \\
\text { calidad según } \\
\text { Norma Rusa }\end{array}$ & Mínimo & Mínimo & NA & NA & NA \\
\hline $\begin{array}{l}\text { Límite de } \\
\text { calidad Norma } \\
\text { Brasil }\end{array}$ & NA & NA & Máximo & Máximo & Máximo \\
\hline
\end{tabular}

\section{Milena Silva Rojas}

Química. Grupo de Investigación Biocalorimetría. Universidad de Pamplona

\section{Alexandra Torres. Ph.D.} Investigador Asociado. Universidad de Pamplona. Director Grupo de Investigación Biocalorimetría.

\section{Wolfgang Hoffmann}

Zootecnista. Grupo de Investigación Biocalorimetría. Universidad de Pamplona 